\title{
Representations of surface groups and right-angled Artin groups in higher rank
}

\author{
STEPHEN WANG
}

\begin{abstract}
We give concrete constructions of discrete and faithful representations of right-angled Artin groups into higher-rank Lie groups. Using the geometry of the associated symmetric spaces and the combinatorics of the groups, we find a general criterion for when discrete and faithful representations exist, and show that the criterion is satisfied in particular cases. There are direct applications towards constructing representations of surface groups into higher-rank Lie groups, and, in particular, into lattices in higher-rank Lie groups.
\end{abstract}

20F36; 53C 35

\section{Introduction}

The study of embeddings of surface groups $\Gamma=\pi_{1}\left(\Sigma_{g}\right)$, where $\Sigma_{g}$ is a closed surface of genus $g$, in Lie groups has a long history, and has been recently the subject of study from a variety of different viewpoints.

The most basic example is when the Lie group $G=\operatorname{PSL}(2, \mathbb{R})$, in which case the faithful, discrete representations inside $\operatorname{Hom}(\Gamma, G) / G$ give Teichmüller space. Hitchin [11] expanded the notion of Teichmüller space to representations into $G=S L(n, \mathbb{R})$, showing in that case that $\operatorname{Hom}(\Gamma, G) / G$ has a distinguished connected component, topologically a ball, containing Teichmüller space. He also calculated the number of connected components in the whole representation space. Other examples include real projective structures on surfaces (see Choi and Goldman [3]), Kähler geometry and bounded cohomology (see Burger, Iozzi and Wienhard [1]), and Anosov flows and hyperconvex curves in projective space (see Labourie [13]).

Looking at the representation variety is also a key component in studying vector bundles over surfaces and surface bundles over surfaces. In addition, when the images of the representations lie in a lattice $\Lambda$ in $G$, one obtains examples of essential surfaces inside a locally symmetric space, generalizing the study of closed geodesics to higher dimensional submanifolds. However, while much has been proven about general properties of $\operatorname{Hom}(\Gamma, G) / G$, such as the number of connected components, or the 
nature of certain types of embeddings, there has not been very much attention paid towards constructing explicit examples of such objects, save in some special cases.

This paper focuses on the general problem of constructing explicit examples of discrete, faithful representations of surface groups into all sorts of Lie groups, or, even better, into lattices. One can then hope to calculate numerical invariants of those representations, such as Toledo invariants (when the associated symmetric space is Hermitian), to determine which components of the representation variety these representations belong to. We apply techniques and results of geometric group theory, combined with an understanding of the geometry of symmetric spaces, to the problem.

The main part of this work is the construction of numerous new explicit examples of discrete, faithful representations of surface groups into semisimple Lie groups of higher rank. We also construct new representations into lattices in semisimple Lie groups. The method developed can also be adapted to representations of other groups of interest in geometric group theory, such as the fundamental groups of closed hyperbolic 3and 4-manifolds (see Crisp-Wiest [5] and Januskiewicz-Świątkowski [12]), and graph braid groups [4].

We examine the topic of representations of surface groups using the technique of right-angled Artin groups. Given a finite graph $H=(V, E)$, we define the right-angled Artin group $A(H)$ to be the group given by the presentation

$$
\left.\left\langle s_{v}\right| v \in V ;\left[s_{v}, s_{w}\right]=1 \text { if } v w \in E\right\rangle .
$$

Despite their simple presentations, these groups exhibit a number of interesting properties, and have been the subject of an increasing amount of study in recent years (see, for example, Charney [2]).

The focus on right-angled Artin groups is useful in studying surface groups because of the work of John Crisp and Bert Wiest [4], who have shown that all fundamental groups of closed, oriented surfaces embed in some right-angled Artin group. In particular, the fundamental group of any closed oriented surface of genus at least 2 embeds into the Artin group $A\left(C_{5}\right)$, where $C_{n}$ denotes the cycle on $n$ vertices.

This is one reason why we primarily focus on Artin groups $A(H)$ where $H$ is a cycle of at least 5 vertices. Another reason is that, since cycles are subgraphs of almost every graph, restrictions on the representations of the corresponding subgroups (or the lack thereof) will translate to information about the full group.

One can show by simple dimension count arguments that representations of $A\left(C_{5}\right)$ exist, but exhibiting faithfulness and, particularly, discreteness requires a stronger argument. Our construction is explicit, and gives a whole class of discrete, faithful 
representations. In particular, the proof of discreteness exploits the geometry of the symmetric spaces associated to Lie groups.

The paper is in two main parts. In the first, we prove that if there is an arrangement of the maximal flats of the symmetric space $X=G / K$ that mimics the structure of the graph $H$ in a certain sense, then we can find a faithful and discrete representation of $A(H)$ into $G$. In the second, we primarily focus on the case where $H$ is a cycle, and show that such arrangements of flats exist for certain symmetric spaces.

This process yields the following main results:

Theorem 1 There are infinitely many conjugacy classes of discrete, faithful representations of $A\left(C_{5}\right)$ into $S L(n, \mathbb{R})$ for $n \geq 3$.

This method ought to be generalizable to arbitrary symmetric spaces, but it sometimes requires Artin groups on larger cycles than $C_{5}$. For certain symmetric spaces, we will not be able to use our method to embed $A\left(C_{5}\right)$, but only Artin groups on cycles of even length. It is known that these Artin groups contain some, but not all, surface groups (see Droms, Servatius and Servatius [6]).

Theorem 2 There are infinitely many conjugacy classes of discrete, faithful representations of $A\left(C_{6}\right)$ into $S O(3,2)$.

It is essential that the Lie groups are of $\mathbb{R}-$ rank at least two.

Theorem 3 If $G$ is a simple Lie group of $\mathbb{R}$-rank 1 , and $H$ is a non-complete connected graph, then there is no discrete and faithful embedding of $A(H)$ into $G$.

It is interesting that this method can also be used to construct representations of surface groups into lattices in semi-simple Lie groups. These constructions yield examples of essential surfaces inside locally symmetric spaces.

Theorem 4 There are infinitely many conjugacy classes of representations of $A\left(C_{5}\right)$ into $S L(5, \mathbb{Z})$.

As a result of Theorem 1, we obtain explicit representations of surface groups.

Theorem 5 For any closed surface group $\Gamma=\pi_{1}\left(\Sigma_{g}\right)$ with $g \geq 2$ and $n \geq 3$, there are infinitely many conjugacy classes of discrete, faithful representations of $\Gamma$ into $S L(n, \mathbb{R})$. 
There is a little bit of work required to keep the infinitely many conjugacy classes, but otherwise this is an immediate corollary. Certain discrete and faithful representations of surface groups into $\operatorname{PSL}(3, \mathbb{R})$ correspond to convex projective structures on the surface, and have been extensively studied (see Choi-Goldman [3] and Goldman [10]). For the most part, however, the techniques used in previous work have not yielded algebraically explicit examples. Our construction gives a way of constructing these representations explicitly.

All examples constructed via this method lie in the connected component of the trivial representation, so these representations do not lie in Hitchin's Teichmüller component or have non-zero Toledo invariant (when the target symmetric space is Hermitian). These are, however, the first class of explicit non-trivial examples of representations, and it is hoped that the geometric nature of the construction (and, in particular, the ease in which it yields discreteness) will be helpful in future study of the representation variety.

\section{Acknowledgements}

I wish to thank Benson Farb, under whose tutelage at the University of Chicago this paper was conceived and (mostly) written. He provided many useful comments and suggestions, and his encouragement and enthusiasm were equally vital. I would also like to thank Dave Morris and Alan Reid for their valuable suggestions, and also Kyle Ormsby for his help with the diagrams.

\section{Preliminaries}

\subsection{Symmetric spaces}

Throughout this paper, $G$ will denote a connected, semisimple Lie group with no compact or Euclidean factors, $K$ a maximal compact subgroup of $G$, and $X=G / K$ its associated symmetric space of non-compact type.

The rank of $X$ is the largest integer $r$ such that there exists a totally geodesic subspace of $X$ isometric to $\mathbb{R}^{r}$. A symmetric space of non-compact type has rank 1 if and only if its sectional curvatures are negative. A maximal flat in a symmetric space $X$ is a totally geodesic submanifold $F$ which is isometric to $\mathbb{R}^{r}$, where $r$ is the rank of $X$. Note that, under the identification of $T_{p} X$ with $\mathfrak{p}, T_{p} F$ is a maximal abelian subalgebra $\mathfrak{a} \subset \mathfrak{p}$.

Every geodesic of $X$ is contained in at least one maximal flat. A geodesic $\gamma$ is called regular if there is exactly one maximal flat containing $\gamma$; if there is more than one 
maximal flats containing $\gamma$, it is singular. Likewise, a point $z \in X(\infty)$ is called regular (resp. singular) if a geodesic $\gamma$ with $\gamma(\infty)=z$ is regular (resp. singular).

An isometry $\phi$ of $X$ having the property that there is some geodesic $\gamma$ such that $\phi(\gamma(t))=\gamma\left(t+t_{0}\right)$ for all $t$ is called axial. In this case, we call $\gamma$ an axis for $\phi$ and $t_{0}$ the translation distance.

For these and other facts on symmetric spaces, see (for instance) Eberlein [9].

\subsection{Artin groups}

Given a finite graph $H=(V, E)$, we define the right-angled Artin group $A(H)$ to be the group given by $\left\langle s_{v}\right| v \in V ;\left[s_{v}, s_{w}\right]=1$ if $\left.v w \in E\right\rangle$.

Crisp and Wiest have shown that all but three surface groups embed in some right-angled Artin group. In particular, for closed orientable surfaces, we have the following:

Theorem 6 (Crisp-Wiest [4]) There is a faithful homomorphism from the fundamental group of any closed oriented surface $\Sigma_{g}$ into the Artin group $A\left(C_{5}\right)$, where $C_{n}$ denotes the cycle on $n$ vertices.

Other Artin groups also contain certain surface groups as subgroups:

Theorem 7 (Droms-Servatius-Servatius [6]) If $n \geq 5$, then $A\left(C_{n}\right)$ contains the surface group $\pi_{1}\left(\Sigma_{g}\right)$, where $g=1+(n-4) 2^{n-3}$.

We will therefore concentrate on the question of finding discrete and faithful representations of right-angled Artin groups, particularly $A\left(C_{5}\right)$, into semi-simple Lie groups.

It will be important that the Lie group be of rank greater than 1 . There are examples of faithful embeddings of $A\left(C_{5}\right)$ into Lie groups of rank 1, but they are far from being discrete.

Theorem 8 If $G$ is a simple Lie group of $\mathbb{R}$-rank 1 , and $H$ is a non-complete connected graph, then there is no discrete and faithful embedding of $A(H)$ into $G$.

Proof Let $v$ and $w$ be two adjacent vertices of $H$, and $s_{v}$ and $s_{w}$ the corresponding generators of the right-angled Artin group. Given a representation $\sigma: A(H) \rightarrow G$, we examine the action of $\sigma\left(s_{v}\right)$ on the symmetric space $X=G / K$, which will be of negative curvature. 
If $\sigma\left(s_{v}\right)$ fixes some point $p \in X$, then $\sigma\left(s_{v}\right)$ lies in a subgroup of $G$ which is compact. Since $s_{v}$ has infinite order in $A(H)$, this means that $\sigma$ is either non-faithful or has non-discrete image.

Thus $\sigma\left(s_{v}\right)$ fixes exactly one or two points in $X(\infty)$. If it fixes two, then there is some geodesic $\gamma: \mathbb{R} \rightarrow X$ and some $t_{0} \in \mathbb{R}$ such that $\sigma\left(s_{v}\right)(\gamma(t))=\gamma\left(t+t_{0}\right)$ for all $t$. This will be the unique geodesic that is fixed by $\sigma\left(s_{v}\right)$, and thus $\gamma$ will be fixed by $\sigma\left(s_{w}\right)$ as well. There will be some $t_{1} \in \mathbb{R}$ such that $\sigma\left(s_{w}\right)(\gamma(t))=\gamma\left(t+t_{1}\right)$ for all $t$.

But this means that, for any $\epsilon>0$, we can find $k, l \in \mathbb{Z}-\{0\}$ such that $\sigma\left(s_{v}^{k} s_{w}^{l}\right)$ translates $\gamma(0)$ a distance less than $\epsilon$ (possibly 0 ), so either $\sigma$ is not faithful or it does not have a discrete image.

We are left with the case where $\sigma\left(s_{v}\right)$ fixes exactly one point $x$ at infinity. The commutation relations then force every element of the image of $\sigma$ to fix $x$, so $\sigma$ is a representation into a horospherical subgroup $N$ of $G$. But $N$ is a nilpotent group (see Eberlein [8]) and is therefore amenable; since $A(H)$ contains a non-abelian free subgroup, it cannot embed faithfully into $N$.

\section{Discrete and faithful representations}

Given a connected graph $H$ with no triangle subgraphs, we will show that the rightangled Artin group $A(H)$ embeds into a higher-rank Lie group $G$ if one can find a configuration of geodesics and flats in the symmetric space $X=G / K$ that mimics the graph $H$. That is to say, if we view the singular geodesics throuigh some point $p_{0} \in X$ as the vertices of a graph $H^{\prime}$, and the maximal flats containing $p_{0}$ as the edges of the graph, we wish to find an induced subgraph of $H^{\prime}$ which is isomorphic to $H$. We will use the terminology of graph theory and say that two geodesics through $p_{0}$ are adjacent if they lie in a common maximal flat.

There will be a few technical conditions, so the exact statement is as follows:

Theorem 9 Let $X=G / K$ be a symmetric space of non-compact type of rank at least 2, and let $p_{0} \in X$. Suppose that for each vertex $v$ of $H$, we can find a geodesic $\gamma_{v}: \mathbb{R} \rightarrow X$ with $\gamma_{v}(0)=p_{0}$ with the following properties:

- If $v w$ is an edge of $H$, then $\gamma_{v}$ and $\gamma_{w}$ are adjacent, there is exactly one maximal flat $F_{v w}$ containing both $\gamma_{v}$ and $\gamma_{w}$, and the set of singular geodesics $S_{v w}$ contained in the copy of $\mathbb{R}^{2} \subset F_{v w}$ determined by $\gamma_{v}$ and $\gamma_{w}$ is finite.

- If $v w$ is not an edge of $H, \gamma_{v}$ and $\gamma_{w}$ are not adjacent. 
- If $v_{1} v_{2}$ and $w_{1} w_{2}$ are disjoint edges in $H$, then no elements of $S_{v_{1} v_{2}}$ and $S_{w_{1} w_{2}}$ are adjacent, with the exception of $\gamma_{v_{i}}$ and $\gamma_{w_{j}}$ if $v_{i} w_{j}$ is an edge of $H$.

- If $v w$ and $w x$ are two edges of $H$ sharing an endpoint, then no element of $S_{v w}-\left\{\gamma_{w}\right\}$ is adjacent to any element of $S_{w x}-\left\{\gamma_{w}\right\}$.

Then there are infinitely many conjugacy classes of discrete, faithful representations $\sigma: A(H) \rightarrow G$.

The proof of the theorem is a modification of the "ping-pong" technique. The classic "ping-pong" argument was first used to prove that two elements $\alpha, \beta$ in $S L(2, \mathbb{R})$ generated a free subgroup. It employed four open sets $U_{\alpha}, U_{\alpha^{-1}}, U_{\beta}, U_{\beta^{-1}} \subset \mathbb{M}^{2}(\infty)$ and showed that a word in $\alpha$ and $\beta$ sent a base point $z \in \mathbb{M}^{2}(\infty)$ to the open set corresponding to the first letter in the word, utilizing the fact that $\alpha$ and $\beta$ had disjoint repelling and attracting fixed sets.

Our proof follows the same spirit, but there are complicating factors due to the fact that $A(H)$ is not free and the more intricate geometry of higher rank symmetric spaces. For instance, our open sets will necessarily intersect one another, and we will be unable to use separate open sets for a generator and its inverse.

We begin the proof with a lemma.

Lemma 10 Let $\mathfrak{g}=\mathfrak{k}+\mathfrak{p}$ be the Cartan decomposition given by a point $p_{0} \in X$, and let $A \in \mathfrak{p}$ be a non-zero vector. Let $F$ denote the union of all maximal flats containing the geodesic $\gamma(t)=\exp (t A) p$. Let $z_{0} \in X(\infty)$; then $\lim _{t \rightarrow \infty} \exp (t A) z_{0}$ will exist, and lie in $F(\infty)$.

Proof Let $z$ be any accumulation point of the sequence $\left\{\exp (t A) z_{0}\right\}$, and let $y=$ $\gamma(-\infty)$. Since $X$ is non-positively curved, the function $f(t)=L_{\gamma(t)}\left(y, z_{0}\right)$ will be non-increasing, and therefore there is some $\beta$ such that $\lim _{t \rightarrow-\infty} f(t)=\beta$.

We claim that $\angle_{\gamma\left(t_{0}\right)}(y, z)=\beta$ for all $t_{0}$. Indeed, for any $\epsilon>0$,

$$
\beta-\epsilon \leq \angle_{\gamma(-t)}\left(y, z_{0}\right) \leq \beta
$$

for $t \gg 0$ so, applying the isometry $\exp \left(\left(t+t_{0}\right) A\right)$, we see that

$$
\beta-\epsilon \leq L_{\gamma\left(t_{0}\right)}\left(y, \exp \left(\left(t+t_{0}\right) A\right) z_{0}\right) \leq \beta
$$

for $t \gg 0$. Hence $\angle_{\gamma\left(t_{0}\right)}(y, z)$ must lie in the interval $[\beta-\epsilon, \beta]$. Since $\epsilon$ was arbitrary, $\angle_{\gamma\left(t_{0}\right)}(y, z)=\beta$, and the claim is proven.

That the claim implies the lemma is precisely Claim E5 in Eberlein [7]. 
Let $\left\{e_{i}\right\}$ denote the set of edges of $H$, and $S_{i}$ the corresponding sets of singular geodesics defined in the statement of the theorem, and denote the unique maximal flat containing the geodesics of $S_{i}$ be denoted by $F_{i}$. Let $S$ be the union of all $S_{i}$, and let $F(\gamma)$ denote the union of all flats containing $\gamma$.

Two geodesics $\gamma, \eta$ are adjacent if and only if $F(\gamma)(\infty)$ and $F(\eta)(\infty)$ are disjoint. Therefore, for each geodesic $\gamma \in S$, we can find an open set $U_{\gamma} \subset X(\infty)$ containing $F(\gamma)(\infty)$ such that $\bar{U}_{\gamma} \cap \bar{U}_{\eta} \neq \varnothing$ iff there is some $i$ such that $\gamma, \eta \in S_{i}$. Let $U_{i}=\bigcap_{\gamma \in S_{i}} U_{\gamma}$; this will be an open set containing $F_{i}(\infty)$.

Let $z \in X(\infty)$ be a point not contained in the closure of any of the $U_{\gamma}$.

Lemma 11 It is possible to find real numbers $t_{v}$ such that the isometries $\phi_{v}=$ $\exp \left(t_{v} A_{v}\right)$ have the following properties:

- If $\gamma \in S$ is not adjacent to $\gamma_{v}$, then $\phi_{v}^{k}\left(U_{\gamma}\right) \subset U_{\gamma_{v}}$ for all $k \in \mathbb{Z}^{\times}$.

- For any $k \in \mathbb{Z}^{\times}, \phi_{v}^{k}(z) \in U_{\gamma_{v}}$.

- If $e_{i}=v w$ is an edge in $H$, and $\gamma \in S$ is adjacent to neither $\gamma_{v}$ nor $\gamma_{w}$, then for any $k, l \in \mathbb{Z}^{\times}, \phi_{v}^{k} \circ \phi_{w}^{l}\left(U_{\gamma}\right) \subset \bigcup_{\eta \in S_{i}} U_{\eta}$.

- If $e_{i}=v w$ is an edge in $H$, then for any $k, l \in \mathbb{Z}^{\times}, \phi_{v}^{k} \circ \phi_{w}^{l}(z) \in \bigcup_{\eta \in S_{i}} U_{\eta}$.

Proof The first two conditions are satisfied as long as the $t_{v}$ are large enough, by the lemma.

To show that the second two are satisfiable, for a given edge $e_{i}=v w$, let $\mathfrak{h} \subset \mathfrak{p}$ be the subspace generated by $A_{v}$ and $A_{w}$, and let $\tau: \mathfrak{h} \rightarrow X(\infty)$ be given by $\tau(A)=$ $\exp (A)\left(z^{\prime}\right)$, for some $z^{\prime} \notin \bigcup_{\eta \in S_{i}} U_{\eta}$. We will show that $\tau^{-1}\left(\bigcup_{\eta \in S_{i}} U_{\eta}\right)$ has a bounded open set in $\mathfrak{h}$ as its complement. This will suffice, for then we can simply pick $t_{v}$ and $t_{w}$ such that $\tau^{-1}\left(\bigcup_{\eta \in S_{i}} U_{\eta}\right) \subset\left\{a A_{v}+b A_{w}|| a \mid>1\right.$ or $\left.|b|>1\right\}$.

To do so, we simply apply the lemma to each geodesic $\exp (t A)\left(p_{0}\right)$, where $A \in \mathfrak{h}$ has length 1 . All but finitely many will be regular geodesics, and for these there is some minimum $T_{A}$ such that $\exp (t A)\left(z^{\prime}\right) \subset U_{i}$ whenever $|t|>T_{A}$. If $\exp (t A)\left(p_{0}\right)=\eta \in S_{i}$, then we can still find some $T_{A}$ such that $\exp (t A)\left(z^{\prime}\right) \subset U_{\eta}$ when $|t|>T_{A}$. Thus along any one-dimensional subspace of $\mathfrak{h}$, the image of $\tau$ will eventually land in $\bigcup_{\eta \in S_{i}} U_{\eta}$. The function $A \rightarrow T_{A}$ will be continuous, and thus there is some $T$ such that $\tau(A) \subset \bigcup_{\eta \in S_{i}} U_{\eta}$ whenever $|A|>T$.

Once we have chosen our $\phi_{v}$, we then define the representation $\sigma: A(H) \rightarrow G$ by $\sigma\left(s_{v}\right)=\phi_{v}$. If $v w$ is an edge of $H$, we know that $\gamma_{v}$ and $\gamma_{w}$ are adjacent, and thus $\phi_{v}$ and $\phi_{w}$ commute. Therefore the relations of $A(H)$ are satisfied, and this is a group 
homomorphism. To show that the image of $\sigma$ is faithful and discrete, we will show that if $h$ is a non-identity element of $A(H)$, then $\sigma(h)(z) \in \bigcup_{\eta \in S_{i}} U_{\eta}$ for some $i$.

For each element $h \in A(H)$, let $\ell(h)$ denote the minimum integer $n$ such that $h$ has a representation $h=s_{v_{1}}^{k_{1}} s_{v_{2}}^{k_{2}} \ldots s_{v_{n}}^{k_{n}}$ as a product of generators of $A(H)$ (where the $k_{i}$ can be either positive or negative integers).

By induction on $n$, we will show that any element $h$ has a representation as a word in the generators $h=s_{v_{1}}^{k_{1}} s_{v_{2}}^{k_{2}} \ldots s_{v_{n}}^{k_{n}}$ where $n=\ell(h)$ and either $\sigma(h)(z) \in U_{v_{1}}$ if $v_{1} v_{2}$ is not an edge of $H$, or $\sigma(h)(z) \in \bigcup_{\eta \in S_{i}} U_{\eta}$ if $v_{1} v_{2}=e_{i}$ is an edge of $H$.

When $\ell(h)=1$, this is true by our choice of the $\phi_{v}$. Now assume that this is true for all $n<m$, and suppose that $\ell(h)=m$. Pick a representation $h=s_{v_{1}}^{k_{1}} s_{v_{2}}^{k_{2}} \ldots s_{v_{m}}^{k_{m}}$. By transposing commuting generators, it might be possible to switch $s_{v_{1}}^{k_{1}}$ to a position farther right; suppose that the farthest right it can go is position $j$, in the word $h=s_{w_{1}}^{l_{1}} s_{w_{2}}^{l_{2}} \ldots s_{w_{m}}^{l_{m}}$. Thus we know that $s_{w_{i}}$ commutes with $s_{w_{j}}$ for all $i<j$, and since there are no triangles in $H, s_{w_{i}}$ does not commute with $s_{w_{i+1}}$ if $i<j-1$.

Also, by our induction hypothesis, we can transpose commuting generators so that the word $h_{j}=s_{w_{j+1}}^{l_{j+1}} \ldots s_{w_{m}}^{l_{m}}$ has the property that $\sigma\left(h_{j}\right)(z)$ is in $U_{w_{j+1}}$ if $w_{j+1} w_{j+2}$ is not an edge of $H$, or $\bigcup_{\eta \in S_{i}} U_{\eta}$ if $w_{j+1} w_{j+2}$ is the edge $e_{i}$ of $H$. We know that $w_{j} w_{j+1}$ cannot be an edge of $H$ (else we could do another transposition to get the original left-most generator $s_{v_{1}}$ farther right). Therefore, if $j=1$, we must have $\sigma(h)(z) \in U_{w_{1}}$, and we are done.

If $j \geq 2$, we see that $\phi_{w_{j-1}}^{l_{j-1}} \circ \phi_{w_{j}}^{l_{j}}$ must take $\sigma\left(h_{j}\right)(z)$ into $\bigcup_{\eta \in S_{i}} U_{\eta}$, where $w_{j-1} w_{j}$ is the edge $e_{i}$ of $H$. If $j=2$, we are then done. If $j>2$, since $w_{i} w_{i+1}$ is not an edge of $H$ for $i<j-1$, we can therefore conclude that $\sigma(h)(z) \in U_{w_{1}}$. This concludes the induction argument.

It remains to be shown that we can get infinitely many conjugacy classes. In the proof of Lemma 11, we were free to choose the $t_{v}$, the translation length of the axial isometries $\phi_{v}$, to be any numbers, as long as they were sufficiently large. Given finitely many discrete and faithful representations $\sigma_{i}$, there are only countably many translation lengths of axial isometries in their images, so as long as we choose a $t_{v}$ distinct from all of them, we are guaranteed a new conjugacy class.

\section{Finding geodesic configurations}

Since the result of Crisp and Wiest holds for a right-angled Artin group on a 5-cycle, we are interested in finding arrangements of 5 singular geodesics that have the properties required by the theorem in the previous chapter. 


\subsection{The model group}

Theorem 12 Let $X=S L(3, \mathbb{R}) / S O(3)$, and $p_{0} \in X$. Then there are infinitely many conjugacy classes of sets of 5 geodesics through $p_{0}$ satisfying the conditions of Theorem 9 for the graph $H=C_{5}$.

Our goal is to find a different flat $F_{12}$ which intersects the original flat $F_{01}$ in one of the singular geodesics $\gamma_{1}$, choose a different singular geodesic $\gamma_{2}$ in $F_{12}$, find another flat $F_{23}$ which intersects $F_{12}$ in $\gamma_{2}$, and so on, until we are able to find a flat $F_{40}$ which intersects $F_{01}$ in a geodesic $\gamma_{0}$, completing the cycle. To find successive flats, we will apply "rotations" around the singular geodesics - that is, isometries fixing the geodesic pointwise - to move one flat to the next (see diagram). We will see that the condition that this process cycle back around to the original flat essentially is a condition on the product of the chosen rotations.

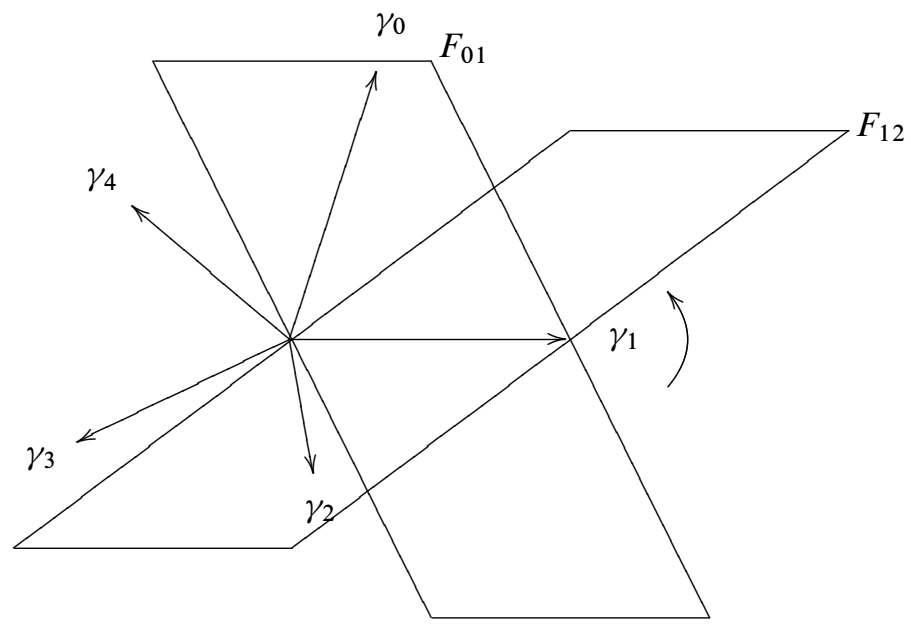

Figure 1: Flats and geodesics in a five-cycle configuration

Without loss of generality, we may take $p_{0}$ to be the identity coset $K=S O(3)$.

Consider the geodesics $\lambda_{1}(t)=\operatorname{diag}\left(e^{-2 t}, e^{t}, e^{t}\right) p_{0}, \lambda_{2}(t)=\operatorname{diag}\left(e^{t}, e^{-2 t}, e^{t}\right) p_{0}$, and $\lambda_{3}(t)=\operatorname{diag}\left(e^{t}, e^{t}, e^{-2 t}\right) p_{0}$. These are all contained in a unique common flat $F_{01}$. Let $A_{i}=\left\{g \in K \mid g \lambda_{i}=\lambda_{i}\right\}$ for each $i$. Note that $A_{1}$ is the set of rotations in $S O$ (3) that fix the $x$-axis, $A_{2}$ fixes the $y$-axis, and $A_{3}$ the $z$-axis.

Pick any non-identity elements $R_{1} \in A_{1}$ and $R_{2} \in A_{2}$. 
Let $Z$ be the vector $(0,0,1)$; consider $Y=R_{2} R_{1} Z$. There is then an $R_{3} \in A_{3}$ such that $R_{3} Y$ has second coordinate equal to 0 , and thus a unique $R_{4} \in A_{1}$ such that $R_{4} R_{3} R_{2} R_{1} Z=Z$.

Let $\gamma_{0}=\lambda_{2}, \gamma_{1}=\lambda_{1}, \gamma_{2}=R_{4} \lambda_{3}, \gamma_{3}=R_{4} R_{3} \lambda_{2}$ and $\gamma_{4}=R_{4} R_{3} R_{2} \lambda_{1}$.

Note that $\gamma_{1}$ and $\gamma_{2}$ both lie in the flat $F_{12}=R_{4} F_{01}$ since $R_{4}$ leaves $\gamma_{1}$ invariant. Similarly, $\gamma_{2}$ and $\gamma_{3}$ both lie in the flat $F_{23}=R_{4} R_{3} F_{01}$, and $\gamma_{3}$ and $\gamma_{4}$ both lie in $F_{34}=R_{4} R_{3} R_{2} F_{01}$. Since $R_{4} R_{3} R_{2} R_{1} \in A_{2}$, both $\gamma_{4}$ and $\gamma_{0}$ are in $F_{40}=$ $R_{4} R_{3} R_{2} R_{1} F_{01}$.

Thus the five geodesics $\gamma_{i}$ satisfy the first condition of Theorem 9. There are finitely many non-adjacency requirements; to verify that geodesics $\eta_{1}$ and $\eta_{2}$ are non-adjacent, we simply need to confirm that axial isometries with $\eta_{1}$ and $\eta_{2}$ as axes do not commute.

There are ten singular geodesics through $p_{0}$ in the five flats, namely the five $\gamma_{i}$, plus $\eta_{0}=\lambda_{3}$ in $F_{01}, \eta_{1}=R_{4} \lambda_{2}$ in $F_{12}, \eta_{2}=R_{4} R_{3} \lambda_{1}$ in $F_{23}, \eta_{3}=R_{4} R_{3} R_{2} \lambda_{3}$ in $F_{34}$, and $\eta_{4}=R_{4} R_{3} R_{2} R_{1} \lambda_{3}$ in $F_{40}$. Let $T_{1}=\operatorname{diag}\left(\frac{1}{4}, 2,2\right), T_{2}=\operatorname{diag}\left(2, \frac{1}{4}, 2\right)$, and $T_{3}=\operatorname{diag}\left(2,2, \frac{1}{4}\right)$. Table 1 gives axial isometries for each of the geodesics.

\begin{tabular}{c|c} 
Geodesic & Isometry \\
\hline$\gamma_{0}$ & $T_{2}$ \\
$\gamma_{1}$ & $T_{1}$ \\
$\gamma_{2}$ & $R_{4} T_{3} R_{4}^{-1}$ \\
$\gamma_{3}$ & $R_{4} R_{3} T_{2} R_{3}^{-1} R_{4}^{-1}$ \\
$\gamma_{4}$ & $R_{4} R_{3} R_{2} T_{1} R_{2}^{-1} R_{3}^{-1} R_{4}^{-1}$ \\
$\eta_{0}$ & $T_{3}$ \\
$\eta_{1}$ & $R_{4} T_{2} R_{4}^{-1}$ \\
$\eta_{2}$ & $R_{4} R_{3} T_{1} R_{3}^{-1} R_{4}^{-1}$ \\
$\eta_{3}$ & $R_{4} R_{3} R_{2} T_{3} R_{2}^{-1} R_{3}^{-1} R_{4}^{-1}$ \\
$\eta_{4}$ & $R_{4} R_{3} R_{2} R_{1} T_{3} R_{1}^{-1} R_{2}^{-1} R_{3}^{-1} R_{4}^{-1}$
\end{tabular}

Table 1: Singular geodesics and axial isometries

Pairs of geodesics that we need to be non-adjacent are $\gamma_{i}$ and $\gamma_{j}$ if $j-1 \neq \pm 1(\bmod$ 5), $\eta_{i}$ and $\eta_{j}$ for any $i \neq j$, and $\eta_{i}$ and $\gamma_{j}$ if $j-i \neq 0,1(\bmod 5)$.

Recall that we have complete freedom to choose $R_{1}$ and $R_{2}$ in $A_{1}$ and $A_{2}$ respectively, but once they are chosen, $R_{3}$ and $R_{4}$ are determined. Since commuting is an analytic condition, if we see that these pairs of isometries do not commute for one particular choice of $R_{1}$ and $R_{2}$, then they will not commute for almost any choice of $R_{1}$ and $R_{2}$. 
We make the choices

$$
R_{1}=\left(\begin{array}{ccc}
1 & 0 & 0 \\
0 & \frac{1}{2} & \frac{\sqrt{3}}{2} \\
0 & -\frac{\sqrt{3}}{2} & \frac{1}{2}
\end{array}\right), R_{2}=\left(\begin{array}{ccc}
\frac{\sqrt{2}}{2} & 0 & \frac{\sqrt{2}}{2} \\
0 & 1 & 0 \\
-\frac{\sqrt{2}}{2} & 0 & \frac{\sqrt{2}}{2}
\end{array}\right) .
$$

This means that $R_{3}$ and $R_{4}$ are the following:

$$
R_{3}=\left(\begin{array}{ccc}
\sqrt{\frac{2}{5}} & \sqrt{\frac{3}{5}} & 0 \\
-\sqrt{\frac{3}{5}} & \sqrt{\frac{2}{5}} & 0 \\
0 & 0 & 1
\end{array}\right), R_{4}=\left(\begin{array}{ccc}
1 & 0 & 0 \\
0 & \sqrt{\frac{5}{8}} & -\sqrt{\frac{3}{8}} \\
0 & \sqrt{\frac{3}{8}} & \sqrt{\frac{5}{8}}
\end{array}\right) .
$$

Verification that the approprate pairs of matrices in Theorem 12 do not commute is done by explicitly calculating their commutators. These are relegated to a separate appendix which is published on the author's web site.

Theorem 13 For any closed hyperbolic surface group $\Gamma$ and $n \geq 3$, there are infinitely many conjugacy classes of discrete, faithful representations of $\Gamma$ into $S L(n, \mathbb{R})$.

The only thing remaining to prove in this theorem is that we can obtain infinitely many conjugacy classes. This is not immediate, since representations of $A\left(C_{5}\right)$ which are not conjugate might still yield representations of a $\pi_{1}(\Sigma)$ which are conjugate. However, the flexibility of our construction proves that you can in fact guarantee different conjugacy classes for the surface groups.

Take any discrete, faithful representation $\sigma: A\left(C_{5}\right) \rightarrow G$ given by our construction. Pick $x \in X=G / K$. Since $\sigma$ is discrete, we know that for any $r>0$, the set $B_{r}=\left\{a \in A\left(C_{5}\right) \mid d(\sigma(a) x, x)<r\right\}$ is finite.

Let $a_{1}, a_{2}, \ldots a_{5}$ be the generators of $A\left(C_{5}\right)$. Then the representation $\sigma_{n}$ given by $\sigma_{n}\left(a_{i}\right)=\sigma\left(a_{i}\right)^{n}$ will be discrete and faithful. The image of $\sigma_{n}$ will be the image of $\sigma$ restricted to the subgroup $A_{n}$ of $A\left(C_{5}\right)$ generated by the $a_{i}^{n}$; we can choose $n$ large enough so that $A_{n} \cap B_{r}=\{e\}$, since $B_{r}$ is finite.

Thus for every $r$, we can find a discrete, faithful representation $\tau: A\left(C_{5}\right) \rightarrow G$ such that $\min \left\{d(x, \tau(a) x) \mid a \in A\left(C_{5}\right)-\{e\}\right\}>r$.

Now suppose that we have a finite set of representations $\tau_{1} \ldots \tau_{n}: \pi_{1}(\Sigma) \rightarrow G$. Take some $a \in \pi_{1}(\Sigma) \subset A\left(C_{5}\right)$ and let $r=\max d\left(x, \tau_{i}(a) x\right)$. If $\tau: A\left(C_{5}\right) \rightarrow G$ is a discrete, faithful representation such that $\min \left\{d(x, \tau(a) x) \mid a \in A\left(C_{5}\right)-\{e\}\right\}>r$, then $\tau$ restricted to $\pi_{1}(\Sigma)$ cannot be conjugate to any of the $\sigma_{i}$. 


\subsection{Other groups}

The example of $S L(3, \mathbb{R})$ serves as a model for embeddings of Artin groups on cycles into other Lie groups of rank 2.

Crisp and Wiest's result about embedding surface groups holds only for $A\left(C_{5}\right)$, so if the cycle is larger than $C_{5}$ we would not be able to embed all surface groups into $G$ via this Artin group technique. However, we would still be able to embed certain surface groups, thanks to the result of Droms, Servatius and Servatius.

For instance, we have the following:

Theorem 14 There are infinitely many conjugacy classes of discrete, faithful representations of $A\left(C_{6}\right)$ and $\pi_{1}\left(\Sigma_{17}\right)$ into $S O(3,2)$.

Here the symmetric space is $X=S O(3,2) / S O(3) \times S O(2)$, again of rank 2 .

Let $p_{0}$ be the identity coset $K=S O(3) \times S O(2)$. We wish to find a collection of six geodesics through $p_{0}$ satisfying the conditions of Theorem 9 .

Let $\mathfrak{a}$ be the abelian subalgebra of $\mathfrak{g}$ generated by the two elements

$$
Y_{0}=\left(\begin{array}{lllll}
0 & 0 & 0 & 1 & 0 \\
0 & 0 & 0 & 0 & 0 \\
0 & 0 & 0 & 0 & 0 \\
1 & 0 & 0 & 0 & 0 \\
0 & 0 & 0 & 0 & 0
\end{array}\right), Y_{1}=\left(\begin{array}{lllll}
0 & 0 & 0 & 0 & 0 \\
0 & 0 & 0 & 0 & 1 \\
0 & 0 & 0 & 0 & 0 \\
0 & 0 & 0 & 0 & 0 \\
0 & 1 & 0 & 0 & 0
\end{array}\right) \text {. }
$$

Then $\exp (\mathfrak{a}) p_{0}$ is a flat $F_{01}$ in $X$, and $\gamma_{0}(t)=\exp \left(t Y_{0}\right) p_{0}$ and $\gamma_{1}(t)=\exp \left(t Y_{1}\right) p_{0}$ are singular geodesics in $F_{01}$ and are perpendicular. The other singular geodesics in $F_{01}$ through $p_{0}$ are the angle bisectors $\lambda_{0}, \lambda_{1}$ of the right angles formed by $\gamma_{0}$ and $\gamma_{1}$. Thus if $T_{0}=\exp \left(a Y_{0}\right)$ and $T_{1}=\exp \left(a Y_{1}\right), T_{0} T_{1}$ and $T_{0} T_{1}^{-1}$ are axial isometries of $X$ whose axes are these singular geodesics $\lambda_{0}$ and $\lambda_{1}$. We choose $a=\log (2+\sqrt{3})$, so

$$
T_{0}=\left(\begin{array}{ccccc}
2 & 0 & 0 & \sqrt{3} & 0 \\
0 & 1 & 0 & 0 & 0 \\
0 & 0 & 1 & 0 & 0 \\
\sqrt{3} & 0 & 0 & 2 & 0 \\
0 & 0 & 0 & 0 & 1
\end{array}\right) ; T_{1}=\left(\begin{array}{ccccc}
1 & 0 & 0 & 0 & 0 \\
0 & 2 & 0 & 0 & \sqrt{3} \\
0 & 0 & 1 & 0 & 0 \\
0 & 0 & 0 & 1 & 0 \\
0 & \sqrt{3} & 0 & 0 & 2
\end{array}\right)
$$

Algebraic $8 \mathcal{G}$ Geometric Topology, Volume 7 (2007) 


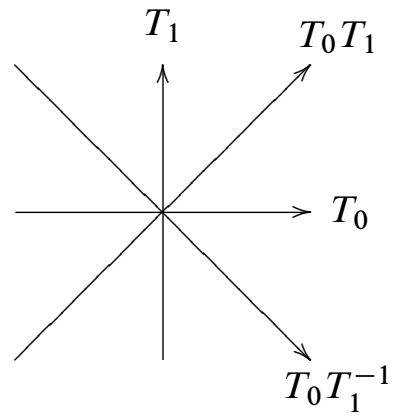

Figure 2: Singular geodesics in a flat in $S O(3,2) / S O(3) \times S O(2)$

Let $A_{0}$ and $A_{1}$ be the subgroups of $K$ fixing $\gamma_{0}$ and $\gamma_{1}$, respectively. There are isomorphisms $\tau_{i}$ between $S O(2)$ and each of the $A_{i}$; they are defined by

$$
\begin{gathered}
\tau_{0}:\left(\begin{array}{cc}
a & b \\
-b & a
\end{array}\right) \in S O(2) \longmapsto\left(\begin{array}{ccccc}
1 & 0 & 0 & 0 & 0 \\
0 & a & b & 0 & 0 \\
0 & -b & a & 0 & 0 \\
0 & 0 & 0 & 1 & 0 \\
0 & 0 & 0 & 0 & 1
\end{array}\right) \in A_{0} \\
\tau_{1}:\left(\begin{array}{cc}
a & b \\
-b & a
\end{array}\right) \in S O(2) \longmapsto\left(\begin{array}{ccccc}
a & 0 & b & 0 & 0 \\
0 & 1 & 0 & 0 & 0 \\
-b & 0 & a & 0 & 0 \\
0 & 0 & 0 & 1 & 0 \\
0 & 0 & 0 & 0 & 1
\end{array}\right) \in A_{1}
\end{gathered}
$$

Copying our proof of Theorem 12, if we can find $R_{1}, R_{3}, R_{5} \in A_{1}$ and $R_{2}, R_{4} \in A_{0}$ such that $R_{5} R_{4} R_{3} R_{2} R_{1} \in A_{0}$, then $\gamma_{0}, \gamma_{1}, \gamma_{2}=R_{5} \gamma_{0}, \gamma_{3}=R_{5} R_{4} \gamma_{1}, \gamma_{4}=$ $R_{5} R_{4} R_{3} \gamma_{0}$ and $\gamma_{5}=R_{5} R_{4} R_{3} R_{2} \gamma_{1}$ are six geodesics which are adjacent in a 6-cycle pattern.

Since $\tau_{0}$ and $\tau_{1}$ have image only in the first factor of $K=S O(3) \times S O(2)$, the condition that $R_{5} R_{4} R_{3} R_{2} R_{1} \in A_{0}$ is essentially just a requirement that a product of rotations in $\mathbb{R}^{3}$ be a rotation around the $x$-axis. Just like in our discussion in the case of $S L(3, \mathbb{R})$, we see that given any choice of $R_{1}, R_{2}, R_{3}$, we know that we can find a rotation $R_{4}$ around the $x$-axis and a rotation $R_{5}$ around the $y$-axis such that the product $R_{5} R_{4} R_{3} R_{2} R_{1}$ fixes the $x$-axis. 
As was the case in Theorem 12, we only need to check non-adjacency for one particular set of choices of $R_{i}$. We choose

$$
R_{1}=\tau_{1}\left(\begin{array}{cc}
\frac{3}{5} & \frac{4}{5} \\
-\frac{4}{5} & \frac{3}{5}
\end{array}\right), \quad R_{2}=\tau_{0}\left(\begin{array}{cc}
\frac{4}{5} & \frac{3}{5} \\
-\frac{3}{5} & \frac{4}{5}
\end{array}\right), \quad R_{3}=\tau_{1}\left(\begin{array}{cc}
-\frac{31}{481} & -\frac{480}{481} \\
\frac{480}{481} & -\frac{31}{481}
\end{array}\right) .
$$

This forces $R_{4}$ and $R_{5}$ to be the following:

$$
R_{4}=\tau_{0}\left(\begin{array}{cc}
\frac{4}{5} & \frac{3}{5} \\
-\frac{3}{5} & \frac{4}{5}
\end{array}\right), \quad R_{5}=\tau_{1}\left(\begin{array}{cc}
\frac{3}{5} & \frac{4}{5} \\
-\frac{4}{5} & \frac{3}{5}
\end{array}\right)
$$

Axial isometries with the $\gamma_{i}$ as axes are $T_{0}, T_{1}, R_{5} T_{0} R_{5}^{-1}, R_{5} R_{4} T_{1} R_{4}^{-1} R_{5}^{-1}$, $R_{5} R_{4} R_{3} T_{0} R_{3}^{-1} R_{4}^{-1} R_{5}^{-1}, R_{5} R_{4} R_{3} R_{2} T_{1} R_{2}^{-1} R_{3}^{-1} R_{4}^{-1} R_{5}^{-1}$. In addition, the extra singular geodesics in the six flats will have axial isometries listed in the following table, where $F_{i j}$ denotes the flat containing $\gamma_{i}$ and $\gamma_{j}$.

\begin{tabular}{c|c} 
Flat & Isometries \\
\hline$F_{01}$ & $T_{0} T_{1}$ \\
& $T_{0} T_{1}^{-1}$ \\
\hline$F_{12}$ & $R_{5} T_{0} T_{1} R_{5}^{-1}$ \\
& $R_{5} T_{0} T_{1}^{-1} R_{5}^{-1}$ \\
\hline$F_{23}$ & $R_{5} R_{4} T_{0} T_{1} R_{4}^{-1} R_{5}^{-1}$ \\
& $R_{5} R_{4} T_{0} T_{1}^{-1} R_{4}^{-1} R_{5}^{-1}$ \\
\hline$F_{34}$ & $R_{5} R_{4} R_{3} T_{0} T_{1} R_{3}^{-1} R_{4}^{-1} R_{5}^{-1}$ \\
& $R_{5} R_{4} R_{3} T_{0} T_{1}^{-1} R_{3}^{-1} R_{4}^{-1} R_{5}^{-1}$ \\
\hline$F_{45}$ & $R_{5} R_{4} R_{3} R_{2} T_{0} T_{1} R_{2}^{-1} R_{3}^{-1} R_{4}^{-1} R_{5}^{-1}$ \\
& $R_{5} R_{4} R_{3} R_{2} T_{0} T_{1}^{-1} R_{2}^{-1} R_{3}^{-1} R_{4}^{-1} R_{5}^{-1}$ \\
\hline$F_{50}$ & $R_{5} R_{4} R_{3} R_{2} R_{1} T_{0} T_{1} R_{1}^{-1} R_{2}^{-1} R_{3}^{-1} R_{4}^{-1} R_{5}^{-1}$ \\
& $R_{5} R_{4} R_{3} R_{2} R_{1} T_{0} T_{1}^{-1} R_{1}^{-1} R_{2}^{-1} R_{3}^{-1} R_{4}^{-1} R_{5}^{-1}$
\end{tabular}

Table 2: Axial isometries by flat

Again, checking the non-adjacency requirements (of which there are 93) is an explicit computation of commutators of these matrices, and is published in a separate appendix on the author's web site.

The proof that there are infinitely many conjugacy classes proceeds in exactly the same way as in Theorem 12.

We have been unable to find a configuration of five maximal flats in $X$ that satisfy all of the requirements of Theorem 9. It is possible to find flats satisfying the first two 
conditions, but in every configuration analyzed, there are always other geodesics in the flats which are adjacent. Changing the Artin group to $A\left(C_{6}\right)$ allows for more freedom in choosing the rotations around each singular geodesic and allows us to avoid this problem.

It seems likely that Artin groups on cycles of 6 or more should be flexible enough to allow representations into most semisimple Lie groups, barring a few obstructions.

For instance, suppose there are only two singular geodesics in a flat through a given point. This is the case when $X=S U(2, n) / S(U(2) \times U(n))$, for instance. In this situation, it will be impossible to embed $A\left(C_{n}\right)$ into the associated Lie group via this method if $n$ is odd, since there will have to be two adjacent geodesics which are conjugates of the same geodesic in the original flat.

Another obstruction occurs for non-irreducible symmetric spaces. If $X$ is a product of rank one symmetric spaces $X_{1} \times X_{2}$, no $A\left(C_{n}\right)$ (for $n>4$ ) will embed faithfully by this method. In this case, if $p_{0}=\left(p_{1}, p_{2}\right)$, singular geodesics through $p_{0}$ are either $\left\{\eta_{1}\right\} \times\left\{p_{2}\right\}$ or $\left\{p_{1}\right\} \times\left\{\eta_{2}\right\}$, where $\eta_{i}$ is a geodesic through $p_{i}$ in $X_{i}$, and any geodesic of the first type will be adjacent to any geodesic of the second, so there is no hope of satisfying the conditions of Theorem 9 for graphs which are not complete and bipartite.

The general conjecture looks like this:

Conjecture 15 If $X=G / K$ is an irreducible symmetric space of rank at least two, and given any maximal flat $F$ and point $p_{0} \in F$, there are at least three singular geodesics in $F$ passing through $p_{0}$, then there are infinitely many conjugacy classes of discrete, faithful representations of $A\left(C_{n}\right)$ into $G$ for any $n \geq 6$.

If $X=G / K$ is an irreducible symmetric space of rank two, and given any maximal flat $F$ and point $p_{0} \in F$, there are exactly two singular geodesics in $F$ passing through $p_{0}$, then there are infinitely many conjugacy classes of discrete, faithful representations of $A\left(C_{2 n}\right)$ into $G$, for any $n \geq 3$.

It would be helpful and interesting to find general methods that would allow for attacking this problem in ways other than a case-by-case analysis.

\section{Lattices}

We can also embed Artin groups inside some lattices in higher rank Lie groups. Recall that a lattice in $G$ is a discrete subgroup $\Gamma \subset G$ such that $\Gamma \backslash G$ has finite volume. Embeddings of surface groups into lattices give examples of essential surfaces inside locally symmetric spaces.

Algebraic 83 Geometric Topology, Volume 7 (2007) 
Theorem 16 There are infinitely many conjugacy classes of representations of $A\left(C_{5}\right)$ into $\Gamma=S L(5, \mathbb{Z})$.

We will find five geodesics to apply the main theorem to.

Let $p_{0}$ be the identity coset in the symmetric space $X=S L(5, \mathbb{R}) / S O(5, \mathbb{R})$. Fix an integer $n \geq 2$, and let $A_{i}$ be the following elements of $\Gamma$ :

$$
\begin{array}{rlrl}
A_{1} & =\left(\begin{array}{ccccc}
n & n-1 & 0 & 0 & 0 \\
n+1 & n & 0 & 0 & 0 \\
0 & 0 & 1 & 0 & 0 \\
0 & 0 & 0 & 1 & 0 \\
0 & 0 & 0 & 0 & 1
\end{array}\right), \\
A_{3} & =\left(\begin{array}{ccccc}
n & 0 & 0 & 0 & n+1 \\
0 & 1 & 0 & 0 & 0 \\
0 & 0 & 1 & 0 & 0 \\
0 & 0 & 0 & 1 & 0 \\
n-1 & 0 & 0 & 0 & n
\end{array}\right), & A_{2}=\left(\begin{array}{ccccc}
1 & 0 & 0 & 0 & 0 \\
0 & 1 & 0 & 0 & 0 \\
0 & 0 & n & n-1 & 0 \\
0 & 0 & n+1 & n & 0 \\
0 & 0 & 0 & 0 & 1
\end{array}\right), \\
A_{5} & =\left(\begin{array}{ccccc}
1 & 0 & 0 & 0 & 0 \\
0 & 1 & 0 & 0 & 0 \\
0 & 0 & 1 & 0 & 0 \\
0 & 0 & 0 & n & n-1 \\
0 & 0 & 0 & n+1 & n
\end{array}\right) . & A_{4}=\left(\begin{array}{ccccc}
1 & 0 & 0 & 0 & 0 \\
0 & n & n-1 & 0 & 0 \\
0 & n+1 & n & 0 & 0 \\
0 & 0 & 0 & 1 & 0 \\
0 & 0 & 0 & 0 & 1
\end{array}\right),
\end{array}
$$

As isometries of $X$, these are all axial hyperbolic isometries. We see easily that $A_{i}$ and $A_{j}$ commute if and only if $i-j \equiv \pm 1(\bmod 5)$, so the geodesics $\gamma_{i}(t)=$ $\exp \left(t \log A_{i}\right) S O(5, \mathbb{R})$ mimic the graph $C_{5}$ in the appropriate way.

The symmetric space is of rank 4 , so the requirement that the set of singular directions in the span of $\log A_{i}$ and $\log A_{j}$ be finite is non-trivial. It is relatively easy to check, however. The maximal flat containing both $\gamma_{1}$ and $\gamma_{2}$ can be taken to the maximal flat $F$ of diagonal matrices via some element $k_{12}$ of $K$ which conjugates $A_{1}$ and $A_{2}$ to the matrices

$$
B_{1}=\left(\begin{array}{ccccc}
\lambda & 0 & 0 & 0 & 0 \\
0 & \lambda^{-1} & 0 & 0 & 0 \\
0 & 0 & 1 & 0 & 0 \\
0 & 0 & 0 & 1 & 0 \\
0 & 0 & 0 & 0 & 1
\end{array}\right), \quad B_{2}=\left(\begin{array}{ccccc}
1 & 0 & 0 & 0 & 0 \\
0 & 1 & 0 & 0 & 0 \\
0 & 0 & \lambda & 0 & 0 \\
0 & 0 & 0 & \lambda^{-1} & 0 \\
0 & 0 & 0 & 0 & 1
\end{array}\right),
$$

where $\lambda$ and $\lambda^{-1}$ are the eigenvalues of the matrix $\left(\begin{array}{c}n \\ n+1\end{array} \begin{array}{l}n-1 \\ n\end{array}\right)$. In the Lie algebra $\mathfrak{a}$ of diagonal traceless matrices, the span corresponds to the subalgebra $\mathfrak{b}$ of all elements 
of the form

$$
\left(\begin{array}{ccccc}
a & 0 & 0 & 0 & 0 \\
0 & -a & 0 & 0 & 0 \\
0 & 0 & b & 0 & 0 \\
0 & 0 & 0 & -b & 0 \\
0 & 0 & 0 & 0 & 0
\end{array}\right) .
$$

Since the only singular directions in $\mathfrak{a}$ are those with equal entries on the diagonal, we see that there are only 4 singular directions in $\mathfrak{b}$, namely those where $a=0, b=0, a=b$, or $a=-b$.

It remains to check the non-adjacency requirements. Let $F_{i j}$ denote the two-plane containing $\gamma_{i}$ and $\gamma_{j}$. Then the singular geodesics through $p_{0}$ in $F_{i j}$ are precisely the axes of the isometries $A_{i}, A_{j}, A_{i} A_{j}$, and $A_{i} A_{j}^{-1}$. There are 125 pairs of geodesics that are supposed to be non-adjacent, but by symmetry arguments we can focus on those involving one geodesic from $F_{12}$ and one geodesic from either $F_{23}$ or $F_{34}$.

Since $A_{1}$ commutes with $A_{2}$ but not with $A_{3}$, it will not commute with either $A_{2} A_{3}$ or $A_{2} A_{3}^{-1}$. Neither $A_{1} A_{2}$ nor $A_{1} A_{2}^{-1}$ commute with $A_{3}$, so they also will not commute with $A_{2} A_{3}$ and $A_{2} A_{3}^{-1}$. Thus all of the pairs of geodesics in $F_{12}$ and $F_{23}$ behave the way they ought.

Checking that no geodesic in $F_{12}$ is adjacent to one in $F_{34}$ (save $\gamma_{2}$ being adjacent $\gamma_{3}$ ) requires a few more explicit matrix calculations, which we relegate to a separate appendix on the author's web site.

Thus the geodesics satisfy the requirements of Theorem 9, and so high enough powers of the $A_{i}$ will give a discrete and faithful subgroup of $S L(5, \mathbb{Z})$ isomorphic to $A\left(C_{5}\right)$.

Note that we could get the image of the representation to lie in any congruence subgroup that we desire, since for any prime $p$, arbitrarily high powers of the $A_{i}$ will be congruent to the identity modulo $p$.

\section{References}

[1] M Burger, A Iozzi, A Wienhard, Surface group representations with maximal Toledo invariant, C. R. Math. Acad. Sci. Paris 336 (2003) 387-390 MR1979350

[2] R Charney, An introduction to right-angled Artin groups preprint

[3] S Choi, W M Goldman, Convex real projective structures on closed surfaces are closed, Proc. Amer. Math. Soc. 118 (1993) 657-661 MR1145415

[4] J Crisp, B Wiest, Embeddings of graph braid and surface groups in right-angled Artin groups and braid groups, Algebr. Geom. Topol. 4 (2004) 439-472 MR2077673 
[5] J Crisp, B Wiest, Quasi-isometrically embedded subgroups of braid and diffeomorphism groups, Trans. Amer. Math. Soc. (to appear)

[6] C Droms, B Servatius, H Servatius, Surface subgroups of graph groups, Proc. Amer. Math. Soc. 106 (1989) 573-578 MR952322

[7] P Eberlein, Geodesic flows on negatively curved manifolds II, Trans. Amer. Math. Soc. 178 (1973) 57-82 MR0314084

[8] P Eberlein, Geometry of 2-step nilpotent groups with a left invariant metric, Ann. Sci. École Norm. Sup. (4) 27 (1994) 611-660 MR1296558

[9] P B Eberlein, Geometry of nonpositively curved manifolds, Chicago Lectures in Mathematics, University of Chicago Press, Chicago, IL (1996) MR1441541

[10] W M Goldman, Convex real projective structures on compact surfaces, J. Differential Geom. 31 (1990) 791-845 MR1053346

[11] N Hitchin, Lie groups and Teichmüller space, Topology 31 (1992) 449-473 MR1174252

[12] T Januszkiewicz, J Świa̧tkowski, Hyperbolic Coxeter groups of large dimension, Comment. Math. Helv. 78 (2003) 555-583 MR1998394

[13] F Labourie, Anosov flows, surface groups and curves in projective space, Invent. Math. 165 (2006) 51-114 MR2221137

Department of Mathematics, Haverford College

Haverford PA 19104, USA

swang@haverford.edu

http://www.haverford.edu/math/swang/

Received: 2 February 2007 Revised: 8 June 2007 